\title{
Avaliação radiográfica da presença de bolhas em restaurações classe II in vitro utilizando diferentes técnicas restauradoras
}

\author{
Radiographic evaluation of the presence of voids in class II in vitro restaurations using \\ different restorative techiniques
}

\author{
Taiane de Oliveira Gonzaga Santos ${ }^{1}$, Tais Rocha Donato², Caroline Mathias ${ }^{3}$, Paula Mathias ${ }^{4 *}$ \\ ${ }^{1}$ Mestranda em Processos Interativos dos Órgãos e Sistemas, Instituto de Ciências da Saúde/UFBA; ${ }^{2}$ Mestre \\ em Processos Interativos dos Órgãos e Sistemas, Instituto de Ciências da Saúde/UFBA; ${ }^{3}$ Doutoranda em Clínica \\ Odontológica - Dentística, Faculdade de Piracicaba/UNICAMP; ${ }^{4}$ Doutora em Clínica Odontológica - Dentística, \\ Faculdade de Piracicaba/UNICAMP
}

\begin{abstract}
Resumo
Introdução: a presença de bolhas no interior das restaurações de resina composta pode reduzir suas propriedades biomecânicas, levando ao insucesso do tratamento. Objetivo: avaliar visual e radiograficamente a presença de bolhas em restaurações de resina composta classe II in vitro utilizando diferentes técnicas restauradoras. Metodologia: foram confeccionados 40 corpos de prova em uma matriz metálica bipartida, randomizados em quatro grupos $(n=10)$ de acordo com a técnica de inserção de resina utilizada: Grupo 1 - resina bulk fill flow e resina regular, Grupo 2 - resina bulk fill regular e resina regular, Grupo 3 - resina regular pela técnica incremental oblíqua e Grupo 4 - resina regular pela técnica incremental horizontal. A avaliação da presença de bolhas foi realizada pelos testes visual e radiográfico. Resultados: o grupo da resina bulk fill flow (G1) foi o que apresentou menor média de bolhas para os dois métodos de avaliação (6,5 para o visual e 2,4 para o radiográfico) e o grupo da técnica incremental horizontal foi o que apresentou maior média de bolhas (24,2 para o teste visual e 4,5 para o radiográfico). Conclusão: a técnica bulk fill apresentou menor formação de bolhas quando comparada à técnica incremental. Desta forma, as resinas bulk fill, principalmente com sua apresentação fluida, parecem ser uma opção viável de material restaurador direto por ser de inserção única, simplificando o procedimento, e evitar a incorporação de bolhas, preservando as propriedades biomecânicas do material.

Palavras-chave: Resinas Compostas. Falha de Restauração Dental. Radiografia Dental.
\end{abstract}

\begin{abstract}
Introduction: the presence of voids within the composite resin restorations can reduce their biomechanical properties, leading to the failure of the treatment. Objetictive: to evaluate visual and radiographically the presence of voids in class II in vitro composite resin restorations using different restorative techniques. Methodology: 40 specimens were made in a bipartite metal matrix, randomized into four groups $(n=10)$ according to the used resin insertion technique: Group 1 - Bulk Fill Flow Resin and Regular Resin, Group 2 -Bulk Fill Regular Resin and Regular Resin, Group 3 - Regular Resin by oblique incremental technique and Group 4-Regular Resin by incremental horizontal technique. The evaluation of the presence of voids was made by visual and radiographic tests. Results: the Bulk Fill Flow Resin group (G1) was that showed lower average of voids for the two evaluation methods (6,5 for the visual test and 2,4 for the radiographic) and the group of horizontal incremental technique was the one that presented higher average of voids (24,2 for the visual test and 4,5 for the radiographic). Conclusion: the Bulk Fill technique showed lower formation of voids compared to incremental technique. This way, the Bulk Fill resins, mainly with their fluid presentation, appear to be a viable option of direct restorative material for being single insertion, simplifying the procedure and preventing the incorporation of air voids, preserving the properties the biomechanical material.

Keywords: Composite Resins. Dental Restoration Failure. Dental Radiography.
\end{abstract}

\section{INTRODUÇÃO}

As resinas compostas têm sido um dos materiais de escolha para restaurações odontológicas, seguindo a linha da odontologia minimamente invasiva. Porém, sua utilização em dentes posteriores ainda apresenta alguns desafios, como deficiência mecânica, alta contração de polimerização e problemas de profundidade de polimerização (TORRES, 2017), os quais impactam diretamente

Correspondente/Corresponding: * Paula Mathias - Faculdade de Odontologia, Universidade Federal da Bahia - End: Av. Araújo Pinho, 72, Canela, Salvador, Ba. CEP: 40.110-912 - Tel: (71) 99116-7275 - E-mail: pmathias@yahoo.com na qualidade e longevidade da restauração.

Existem evidências de que o estresse resultante da contração de polimerização pode promover infiltração marginal, deflexão de cúspide, trincas dentais, redução na resistência de união, formação de gaps e cáries secundárias (ALMEIDA JUNIOR, 2017).

$\mathrm{Na}$ tentativa de reduzir esses efeitos deletérios, utiliza-se a inserção incremental da resina composta, que faz com que ela se ligue a um número reduzido de paredes da cavidade, resultando em menor tensão marginal (ALMEIDA JUNIOR, 2017; CASANOVA et al., 2002; COSTA, 2016; TORRES, 2017). Essa técnica consiste na 
colocação de incrementos de resina composta de até 2 $\mathrm{mm}$ de espessura, polimerizados separadamente, para se obter uma completa polimerização e reduzir a contração. (ALQUDAIHI, et al., 2018; COSTA, 2016; TORRES, 2017). As camadas de resina podem ser inseridas na cavidade de forma horizontal ou oblíqua, que resulta em uma melhor distribuição de tensões (ROSATTO, 2015).

Entretanto, essa técnica possui alguns inconvenientes como a demanda de maior tempo para execução da restauração, a possibilidade de incorporação de bolhas de ar e contaminação por umidade, devido à dificuldade de condensação adequada entre os incrementos (ALQUDAIHI, et al., 2018; CHAVES, 2017; COSTA, 2016; SOARES et al., 2017; TORRES, 2017).

A presença de bolhas no interior da restauração é prejudicial à performance biomecânica das resinas compostas, podendo acelerar o processo de deterioração do material. Quando presentes nas margens, as bolhas podem levar à microinfiltração e descoloração; entre os sucessivos incrementos geram menor resistência à flexão e maior desgaste; e na superfície da restauração há necessidade de tempo extra de atendimento para removê-las (ALMEIDA JUNIOR, 2017; SOARES et al., 2017; NAZARI et al., 2013).

A incorporação de bolhas pode ocorrer durante o processo de fabricação do material, durante o manuseio na inserção da resina ou ser relacionada à sua viscosidade durante a colocação e à técnica de inserção. Resinas compostas com maior viscosidade tendem a gerar mais bolhas que materiais de baixa viscosidade (ALMEIDA JUNIOR, 2017; ALQUDAIHI, et al., 2018;).

Para superar as limitações dos materiais restauradores e melhorar as propriedades mecânicas, foram desenvolvidas as resinas bulk fill, (SOARES et al., 2017). Elas podem ser colocadas em incrementos únicos de 4 $\mathrm{mm}$ a $5 \mathrm{~mm}$ devido a evolução das unidades fotoiniciadoras, às suas características monoméricas e pela sua translucidez elevada, que permite maior penetração da luz em maior profundidade (ALQUDAIHI, et al., 2018; CHAVES, 2017). Geram menor estresse de contração e menor deformação de cúspide que a resina convencional, com uma técnica mais simples e rápida (ALQUDAIHI et al., 2018; LINS, 2017).

A fim de avaliar os possíveis defeitos no interior dos compósitos, métodos não destrutivos têm sido usados, como a radiografia digital, microtomografia computadorizada e, mais recentemente a tomografia de coerência óptica (HAN et al., 2016; NAZARI et al., 2013). Estes últimos, apesar de serem superiores no que se refere a qualidade da imagem, não são muito acessíveis na rotina clínica como a radiografia.

A radiografia digital pode ser usada na verificação de defeitos internos em restaurações odontológicas e possui diversas vantagens quando comparada à radiografia analógica, como maior eficácia na detecção de bolhas e outros defeitos internos nas resinas compostas. Além de requerer menor radiação, não utiliza químicos tóxicos e apresenta custo menor ao longo do tempo (NIEMIEC, 2007; RODRIGUES et al., 2014).

Dessa forma, este estudo teve como objetivo avaliar, visual e radiograficamente, a presença de bolhas em restaurações Classe II in vitro, utilizando quatro diferentes técnicas restauradoras e materiais resinosos.

\section{METODOLOGIA}

Foram confeccionados, por um único operador, 40 corpos de prova em uma matriz retangular metálica bipartida ( $4 \mathrm{~mm} \times 6 \mathrm{~mm} \times 4 \mathrm{~mm}$ ), randomizados em 4 grupos $(n=10)$ de acordo com o material e a técnica restauradora utilizada:

- Grupo $1=4 \mathrm{~mm}$ de Resina Bulk Fill Flow (BFF) + $2 \mathrm{~mm}$ de Resina Regular (RR);

- Grupo $2=4 \mathrm{~mm}$ de Resina Bulk Fill Regular (BFR) $+2 \mathrm{~mm}$ de Resina Regular (RR);

- Grupo 3 = Resina Regular (RR) com incrementos oblíquos de $2 \mathrm{~mm}$ cada;

- Grupo 4 = Resina Regular (RR) com incrementos horizontais de $2 \mathrm{~mm}$ cada.

A Figura 1 mostra a representação esquemática dos grupos experimentais, e os materiais utilizados estão listados na Tabela 1.

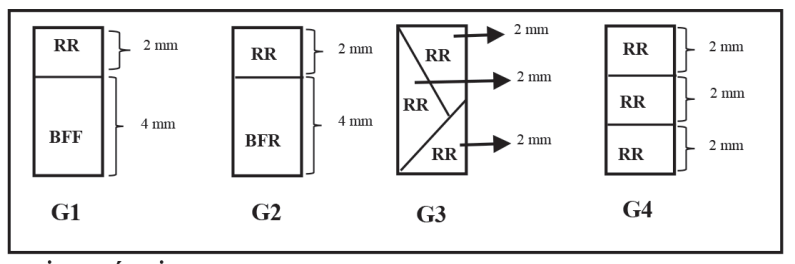

Autoria própria

Tabela 1 - Materiais utilizados, composição, instruções do fabricante.

\begin{tabular}{|c|c|c|}
\hline Material & Composição & Instruções do fabricante \\
\hline $\begin{array}{l}\text { Resina Opus Bulk Fill Flow - A1 } \\
\text { (FGM) }\end{array}$ & TEGDMA, Bis-EMA, canforoquinona, coiniciador. & $\begin{array}{l}\text { Aplicação através da ponteira de incrementos de até } 4 \mathrm{~mm} \text {. Fotopolimerização } \\
\text { por } 20 \mathrm{seg} \text {, mínimo de potência de } 450 \mathrm{~mW} / \mathrm{cm}^{2} \text {. }\end{array}$ \\
\hline $\begin{array}{l}\text { Resina Opus Bulk Fill - A2 } \\
\text { (FGM) }\end{array}$ & TEGDMA, Bis-EMA, canforoquinona, coiniciador. & $\begin{array}{l}\text { Aplicação com espátula de incrementos de até } 5 \mathrm{~mm} \text {. Fotopolimerizar por } 40 \mathrm{seg} \text {, } \\
\text { com potência mínima de } 450-1000 \mathrm{~mW} / \mathrm{cm}^{2}\end{array}$ \\
\hline $\begin{array}{l}\text { Resina Opallis - A1, A2 e A4 } \\
\text { (FGM) }\end{array}$ & $\begin{array}{l}\text { Monômeros Bis (GMA), Bis (EMA), UDMA e } \\
\text { TEGDMA, canforoquinona, coiniciador e silano. }\end{array}$ & $\begin{array}{l}\text { Técnica de inserção e polimerização por incremento. Fotopolimerização por } 20 \\
\text { seg, com potência mínima de } 450 \mathrm{~mW} / \mathrm{cm}^{2}\end{array}$ \\
\hline
\end{tabular}


A espessura dos incrementos foi medida com uma sonda periodontal milimetrada (Trinity). Diferentes cores de resina foram utilizadas nos incrementos para diferenciar o topo da base dos corpos de prova (A1 na base e A2 no topo para o G1, A2 na base e A4 no topo para o grupo G2, A2 na base, A4 intermediário e A1 no topo para os grupos $\mathrm{G} 3$ e G4).

Os incrementos foram colocados na matriz metálica com o auxílio de uma espátula de inserção de resina (Suprafill no 1, Duflex), exceto para a resina Bulk Fill Flow, cuja inserção na matriz foi realizada com a própria ponteira de aplicação da resina. Cada incremento de $4 \mathrm{~mm}$ foi fotopolimerizado por 40 segundos, e os de $2 \mathrm{~mm}$, por 20 segundos, com fotopolimerizador de luz LED (VALO, Ultradent Products, Inc) com intensidade de luz de 1000 $\mathrm{mW} / \mathrm{cm}^{2}$. Os corpos de prova foram, então, identificados e armazenados individualmente em recipientes com 2 $\mathrm{ml}$ de água destilada e mantidos em estufa à $37^{\circ} \mathrm{C}$. Todas as faces dos corpos de prova foram polidas com discos abrasivos Soflex (3M), em baixa rotação, na sequência de granulação grosso, médio e fino.

Para a avaliação da presença de bolhas, foi realizada avaliação visual e radiográfica dos corpos de prova. $\mathrm{Na}$ avaliação visual, um examinador treinado observou, com auxílio de uma lupa, com aumento de $5 x$, a presença de bolhas nos corpos de prova, as quais foram contabilizadas por face (base, topo e quatro faces circundantes), gerando um total de bolhas por grupo.

$\mathrm{Na}$ avaliação radiográfica, cada corpo de prova foi radiografado por aparelho de Raio-x odontológico com sensor digital (Sensor Digital Micro Imagem FIT - T2) e exposição de 0,16 segundos, padronizando-se a posição dos corpos de prova com um posicionador radiográfico. As imagens obtidas no programa foram analisadas por um examinador treinado quanto à presença de imagens circulares radiolúcidas no interior dos corpos de prova, as quais foram quantificadas, gerando um total para cada grupo.

Os dados foram tabulados no Excel 2016. A análise descritiva foi realizada pelo cálculo da média e do desvio-padrão dos grupos. Para a análise inferencial dos dados realizou-se uma análise de variância com 1 fator (ANOVA
1-Fator) para identificar se havia diferença estatística entre os grupos, seguida do post hoc de Tukey. Os pressupostos da ANOVA foram confirmados com o teste de Levene e teste de Shapiro-Wilk, que indicaram, respectivamente homocedasticidade e normalidade dos resíduos. A correlação de Pearson foi utilizada entre a inspeção visual e a radiográfica. Foi adotado nível de significância de $95 \%$ e $p=0,05$.

\section{RESULTADOS}

A Tabela 2 apresenta a média e o desvio-padrão da quantidade de bolhas detectadas pela inspeção visual e pela radiográfica para os quatro grupos de estudo.

Tabela 2 - Média \pm DP da quantidade de bolhas para avaliação visual e radiográfica por grupo.

\begin{tabular}{lcc}
\hline Grupo & $\begin{array}{l}\text { Avaliação visual } \\
\text { (Média } \pm \text { Desvio-padrão) }\end{array}$ & $\begin{array}{l}\text { Avaliação radiográfica } \\
\text { (Média } \pm \text { Desvio-padrão) }\end{array}$ \\
\hline G1 & $6,5 \pm 2,37^{\mathrm{c}}$ & $2,4 \pm 0,84^{\mathrm{b}}$ \\
\hline $\mathrm{G} 2$ & $12,9 \pm 3,35^{\mathrm{b}}$ & $3,5 \pm 1,18^{\mathrm{ab}}$ \\
\hline $\mathrm{G} 3$ & $20,6 \pm 4,03^{\mathrm{a}}$ & $4,6 \pm 0,7^{\mathrm{a}}$ \\
\hline $\mathrm{G} 4$ & $24,2 \pm 2,57^{\mathrm{a}}$ & $4,5 \pm 1,78^{\mathrm{a}}$ \\
\hline
\end{tabular}

Letras sobrescritas diferentes indicam diferença estatística entre os grupos. ANOVA 1-Fator / Teste de Tukey. Autoria própria

Na inspeção visual, o grupo cuja inserção da resina foi realizada pela técnica incremental horizontal (G4) foi o que apresentou maior média de bolhas, seguido do da técnica incremental oblíqua (G3). Houve diferença estatística entre os grupos, exceto entre os grupos G3 e G4. O grupo da resina bulk fill flow (G1) foi o que apresentou menor quantidade e bolhas.

Para a inspeção radiográfica, os grupos G3 e G4 apresentaram maior média da quantidade de bolhas, sem haver diferença estatística entre eles. Houve diferença estatística entre os grupos G1 e G3, G1 e G4. O grupo que apresentou menor quantidade de bolhas foi o $\mathrm{G} 1$ e o que teve maior quantidade foi o G3.

A figura 2 mostra as radiografias obtidas dos corpos de prova representativos de cada grupo.

Figura 2 - Radiografias digitais representativas dos quatro grupos.

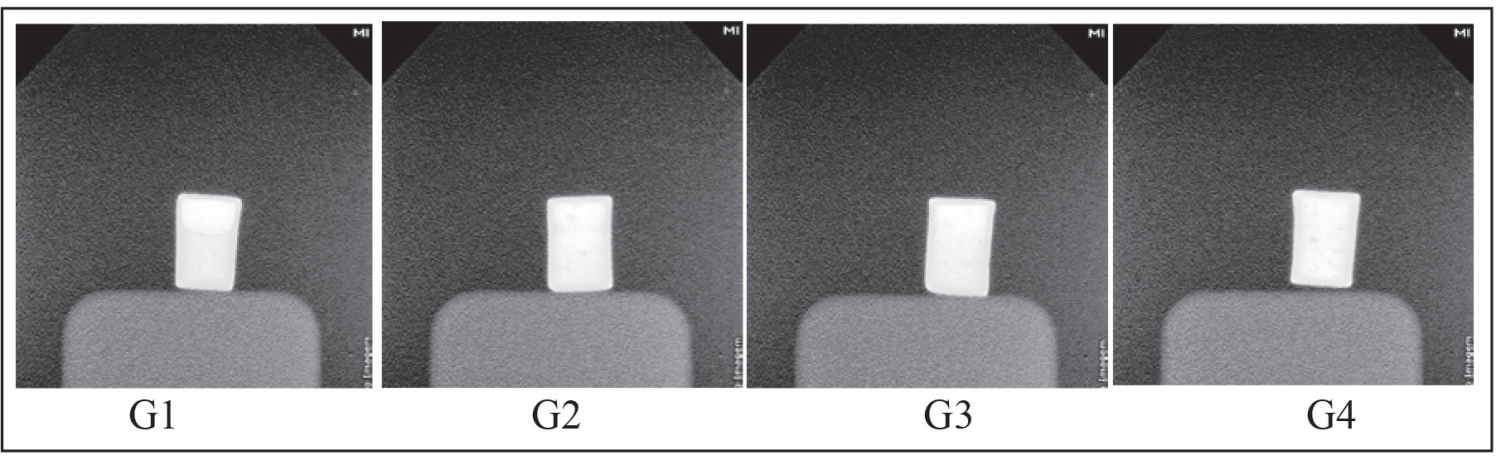


Autoria própria

A correlação de Pearson entre a avaliação visual e a radiográfica mostrou correlação estatisticamente significante entre os testes $(p<0,01)$. O coeficiente de correlação foi de 0,63 , mostrando uma correlação positiva, entre moderada e alta para as duas avaliações. O coeficiente de determinação $r^{2}=0,397$, indica que $39,7 \%$ dos valores de um tipo de inspeção explicam os achados da outra.

A Figura 3 mostra que a análise radiográfica possui baixa sensibilidade para detecção de bolhas.

Figura 3 - Gráfico de dispersão entre inspeção visual e radiográfica.

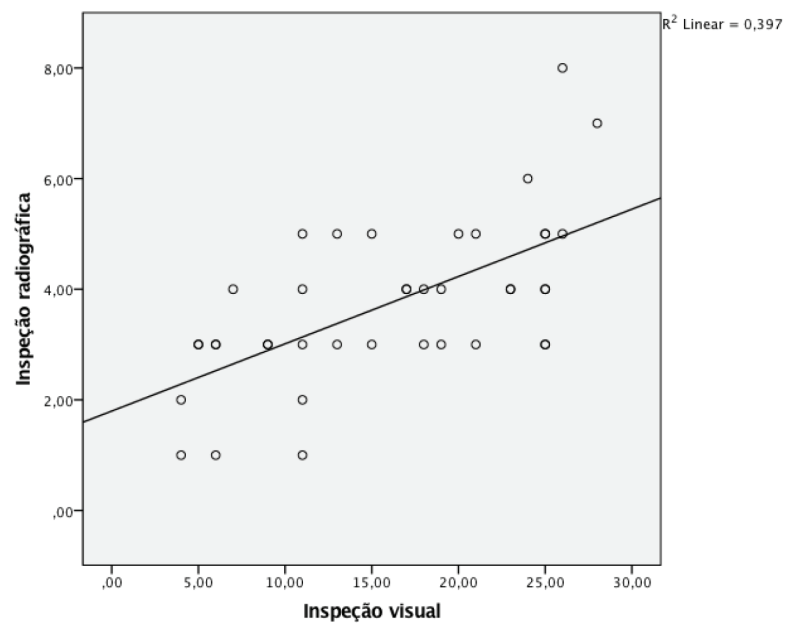

Autoria própria

\section{DISCUSSÃO}

A presença de bolhas no interior da restauração de resina composta pode levar ao insucesso desse procedimento. Isso ocorre devido a falhas como microinfiltração, manchamento, redução na resistência à fadiga e ao desgaste ao redor da bolha e diminuição da força flexural quando ela está presente entre os incrementos de resina composta. Além disso, as bolhas podem aparecer como imagens radiolúcidas nas radiografias, podendo ser mal interpretadas como lesões de cárie secundárias, especialmente quando estão entre o material restaurador e o dente, levando a substituições desnecessárias de restauração (ALMEIDA JUNIOR, 2017; NAZARI et al.,2013; SOARES et al., 2017).

Os grupos com maior média de bolhas para as duas avaliações foram os da resina convencional, que utilizaram as técnicas incrementais oblíqua e horizontal (G3 com média de 20,6 para inspeção visual e 4,6 para inspeção radiográfica, G4 com média de bolhas na inspeção visual de 24,2 e 4,5 na inspeção radiográfica), sem diferença estatística entre eles. Esses resultados podem ser explicados pelo fato de a inserção incremental poder gerar mais chances de incorporar bolhas entre os incrementos (PARK et al., 2008), o que pode ser reduzido quando a inserção da resina é única, como quando se utiliza a resina bulk fill.

Entretanto, Almeida Junior (2017), avaliou a presença de bolhas em restaurações de resinas compostas convencionais e tipo bulk fill por microtomografia computadorizada e concluiu que a inserção da resina convencional pela técnica incremental resultou na redução do número de bolhas no corpo do material quando comparada à técnica sônica. Esse resultado pode ser explicado pelo fato de que a espatulação reduziria o número de bolhas ou outros defeitos presentes na matriz durante a manipulação do material.

Em 2017, Alqudaihi et al. também encontraram melhor adaptação interna com a resina convencional colocada de forma incremental, comparada aos materiais bulk fill. O grupo com menor incidência de bolhas foi o que utilizou a resina SonicFill. A resina composta com maior viscosidade apresentou maior probabilidade de gerar bolhas do que os materiais de baixa viscosidade, pelo fato de o preenchimento dos compósitos poder apresentar bolhas do processo de fabricação, sendo difícil para uma matriz de alta viscosidade penetrá-las.

No presente estudo, o grupo da resina bulk fill flow foi o que apresentou menor média de bolhas. Esse resultado pode ter ocorrido devido ao processo de fabricação ter fornecido melhores condições ao material e à habilidade do operador em manipulá-lo.

Soares et al. (2017) analisaram a detecção de bolhas na restauração com resinas convencionais colocadas em incrementos oblíquos, uma bulk fill de alta viscosidade, colocada em uma única camada, e compósitos fluídos, colocados em duas camadas. Concluíram que a localização das bolhas em compósitos bulk fill tende a se concentrar nos ângulos gengivais e axiais. Muitas bolhas foram observadas entre a camada de compósito fluido e a camada de compósito convencional usada. Para a resina convencional, as bolhas coincidiram com a região entre os incrementos. Linhas de fratura foram observadas nessas regiões, sugerindo que a presença de bolhas pode reduzir a força coesiva da restauração.

Da mesma forma, neste estudo, os grupos da resina convencional em incrementos apresentaram mais bolhas, muitas vezes coincidindo com as regiões entre os incrementos. O mesmo achado foi descrito por Rosatto (2015) que avaliou radiograficamente o efeito da restauração com resina bulk fill em comparação com a técnica incremental da resina convencional. A inserção de muitos incrementos pode resultar na incorporação de bolhas devido à dificuldade de condensação adequada dos incrementos.

Os testes utilizados neste estudo para avaliar a presença de bolhas no interior das resinas foram o teste visual direto e o radiográfico digital. Ambos são classificados como testes não-destrutivos, nos quais se faz a inspeção de falhas nos materiais sem cortar ou alterar o objeto de estudo (GHOLIZADEH, 2016; HAN et al, 2016). O teste radiográfico se mostrou menos sensível para a detecção de 
bolhas do que o teste visual. Porém, em situações clínicas, o teste visual não pode ser realizado, então a radiografia digital pode ser um bom método de avaliação de defeitos no interior das restaurações.

\section{CONCLUSÕES}

A resina bulk fill flow foi a que mostrou menor presença de bolhas na avaliação visual. 0 uso de resinas convencionais usando as técnicas incrementais resultou na presença de maior quantidade de bolhas, analisadas pelo método visual. Dessa forma, a técnica bulk fill, principalmente com sua apresentação fluida, parece ser uma opção viável de material restaurador direto para simplificar o procedimento, por ser de inserção única e evitar a incorporação de bolhas. Quanto à detecção dessas bolhas, o teste radiográfico mostrou menor sensibilidade que o teste visual. Porém, ainda assim, foi capaz de detectá-las, sendo uma opção válida e acessível ao cirurgião-dentista.

\section{REFERÊNCIAS}

ALMEIDA JUNIOR, L. J.dos S. Estudo do comportamento de resinas bulk fill em restaurações classe I por meio de microtomografia computadorizada e microtração. 2017. 77f. Tese (Doutorado Programa de Pós-Graduação em Odontologia) - Universidade Federal do Maranhão, São Luís, 2017.

ALQUDAIHI, F. S. et al. A. Comparison of internal adaptation of bulk-fill and increment-fill resin composite materials. Oper. Dent., 2018. DOI: 10.2341/17-269-L.

CASANOVA, R. C. et al. Influência das técnicas de inserção de resina composta condensável sobre a infiltração marginal. Cienc. Odontol. Bras., São Paulo, v. 5, n. 3, p. 62-9, set./dez. 2002.

CHAVES, L. V. de F. Compósitos bulk fill fluídos versus resinas compostas tradicionais: comportamento mecânico. 2017. 41f. Dissertação (Mestrado em Saúde Coletiva) - Universidade Federal do Rio Grande do Norte, Natal, 2017.
COSTA, T. R. F. Avaliação clínica e laboratorial de restaurações com resina Bulk Fill inseridas de forma incremental ou em incremento único. 2016. 136f. Tese (Doutorado em Dentística Restauradora) - Universidade Estadual de Ponta Grossa, Ponta Grossa, 2016.

GHOLIZADEH, S. A review of non-destructive testing methods of composite materials. Procedia Structural Integrity, [S.I], v. 1, p. 050-057, 2016.

HAN, S. H. et al. Non-destructive evaluation of an internal adaptation of resin composite restoration with swept-source optical coherence tomography and micro-CT. Dent. mater., Washington, v. 32, p e1-e7, 2016.

LINS, R. B. E. Influência da técnica e material restaurador no comportamento biomecânico em Restaurações Classe II. 2017. 47f. Dissertação mestrado - Universidade Estadual de Campinas/Faculdade de Odontologia de Piracicaba, Piracicaba, 2017.

NAZARI, A. et al. Non-destructive characterization of voids in six flowable composites using swept-source optical coherence tomography. Dent. mater., Washington, v. 29, p 278-286, 2013.

NIEMIEC, B. A. Digital dental radiography. J. vet. dent., Boise, v. 24, n. 3, 2007.

PARK, J. et al. How should composite be layered to reduce shrinkage stress: incremental or bulk filling? Dent. mater., Washington v. $24, n$. 11, p. 1501-1505, 2008.

RODRIGUES, F. C. et al. Avaliação de dois métodos radiográficos (analógico e digital) na deteç̧ão de bolhas em resina composta in vitro. Braz. J. Surg. Clin. Res., Paraná, v. 5, n. 3, p. 33-36, 2014.

ROSATTO, C. M. P. de. Restaurações classe II MOD em molares empregando resinas compostas de incremento único e técnica incremental - análise biomecânica experimental e computacional. 2015. 106f. Dissertação (Mestrado em Odontologia) - Universidade Federal de Uberlândia, Uberlândia, 2015.

SOARES, C. J. et al. Radiopacity and porosity of Bulk Fill and conventional composite posterior restorations - Digital X-ray analysis. Oper. Dent., Minas Gerais, v. 42, n. 6, p 616-625, 2017. DOI: 10.2341/16-146 L.

TORRES, A. E. Resina bulk fill: relato de caso clínico. 2017. 27f. TCC (Especialização em Dentística Restauradora) - Universidade Federal do Rio Grande do Sul, Porto Alegre, 2017.

Submetido em:14/11/2018

Aceito em: 29/11/2018 